\title{
Could bow-shaped magnetic morphologies surround filamentary molecular clouds?
}

\section{The 3D magnetic field structure of Orion-A}

\author{
M. Tahani ${ }^{1,2}$, R. Plume ${ }^{2}$, J. C. Brown ${ }^{2}$, J. D. Soler ${ }^{3}$, and J. Kainulainen ${ }^{3,4}$ \\ ${ }^{1}$ Dominion Radio Astrophysical Observatory, Herzberg Astronomy and Astrophysics Research Centre, National Research Council \\ Canada, PO Box 248, Penticton, BC V2A 6J9, Canada \\ e-mail: mtahani@ucalgary.ca \\ 2 Physics \& Astronomy, University of Calgary, Calgary, Alberta, Canada \\ ${ }^{3}$ Max-Planck-Institute for Astronomy, Königstuhl 17, 69117 Heidelberg, Germany \\ ${ }^{4}$ Chalmers University of Technology, Department of Space, Earth and Environment, 41293 Gothenburg, Sweden
}

Received 9 July 2019 / Accepted 17 October 2019

\begin{abstract}
Context. A new method based on Faraday rotation measurements recently found the line-of-sight component of magnetic fields in Orion-A and showed that their direction changes from the eastern side of this filamentary structure to its western side. Three possible magnetic field morphologies that can explain this reversal across the Orion-A region are toroidal, helical, and bow-shaped morphologies.

Aims. In this paper, we constructed simple models to represent these three morphologies and compared them with the available observational data to find the most probable morphology(ies).

Methods. We compared the observations with the models and used probability values and a Monte Carlo analysis to determine the most likely magnetic field morphology among these three morphologies.

Results. We found that the bow morphology had the highest probability values, and that our Monte-Carlo analysis suggested that the bow morphology was more likely.

Conclusions. We suggest that the bow morphology is the most likely and the most natural of the three morphologies that could explain a magnetic field reversal across the Orion-A filamentary structure (i.e., bow, helical and toroidal morphologies).
\end{abstract}

Key words. magnetic fields - stars: formation - ISM: clouds - ISM: magnetic fields

\section{Introduction}

Theoretical studies suggest that the orientation and strength of magnetic fields are dynamically important in the formation and evolution of filaments and filamentary molecular clouds (MC; e.g., Hartmann et al. 2001; McKee \& Ostriker 2007; Hennebelle \& Falgarone 2012; Seifried \& Walch 2015; Hennebelle \& Inutsuka 2019). Observations of magnetic fields indicate that there is a coupling between the matter and the magnetic fields in these regions (e.g., Crutcher 2012; Li et al. 2013; Planck Collaboration XXXV 2016). However, these studies are not conclusive, and we require more studies, techniques, and observations to constrain the role and effects of magnetic fields in these star-forming regions.

To explore magnetic field morphologies, a number of observations have been done exploiting dust polarization and Zeeman measurements. For example, the Planck Collaboration observed the plane-of-sky component of the magnetic field $\left(B_{\perp}\right)$ in different MCs (Planck Collaboration XXXV 2016, hereafter PXXXV). Zeeman measurements have been successful in obtaining the magnetic field component parallel to the line of sight $\left(\mathrm{B}_{\|}\right)$in star-forming regions (e.g., Goodman et al. 1989; Crutcher 1999). Troland \& Crutcher (2008) report the largest number of $\mathrm{OH}$ Zeeman observations toward MCs. A recent study by Tahani et al. (2018, hereafter TPBK18) proposes a new method based on Faraday rotation to determine $\mathrm{B}_{\|}$in MCs. Prior to the TPBK18 method, however, Faraday rotation studies were traditionally associated with the large scale Galactic magnetic field and mostly ionized medium.

Each of these observations only provides one component of the magnetic fields, meaning, $\mathrm{B}_{\|}$or $\mathrm{B}_{\perp}$. Probing the threedimensional (3D) field morphology requires combining the individual measurements (Zeeman splitting, Faraday rotation, and dust polarization technique), developing a new technique, or using geometrical models. Recently, Chen et al. (2019) proposed obtaining the 3D magnetic field morphology using dust polarization observations, based on the statistical properties of the observed polarization fraction. Another technique to obtain the 3D magnetic field in star-forming regions involves combining dust polarization data, Zeeman measurements, and ion-toneutral molecular line width ratio measurements (e.g. Houde et al. 2002). However, due to a degeneracy between the inclination angle and the strength of $\mathrm{B}_{\perp}$, and ambiguity in the direction of $\mathrm{B}_{\perp}$, Li \& Houde (2008) and Houde (2011) suggested that this technique cannot be widely used. Geometrical models have also been used to provide insights to the 3D magnetic field morphologies of filamentary structures (e.g. Planck Collaboration XXXII 2016; Planck Collaboration XXXIII 2016). The method of TPBK18 holds the promise of wider exploration of $B_{\|}$, with future RM surveys with more sensitive measurements 
and reduced uncertainties. This will improve our understanding of the 3D magnetic field morphologies associated with star forming MCs.

TPBK18 found $\mathrm{B}_{\|}$in Orion-A, Orion-B, California, and Perseus MCs. They found that, within the uncertainties, their obtained values are consistent with the available molecular Zeeman measurements in these regions. They revealed that in California and Orion-A MCs, the direction of line-of-sight (LOS) magnetic fields reverses from one side of these filamentary structures to the other side. This magnetic field reversal in Orion-A was previously observed (Heiles 1997; Heiles \& Robishaw 2009) and has been interpreted in different ways.

Three magnetic field morphologies that can explain this direction-change of $\mathrm{B}_{\|}$across filamentary structures are toroidal, helical, and bow morphologies. The helical or toroidal morphologies have been investigated in a number of theoretical studies (e.g., Shibata \& Matsumoto 1991; Nakamura et al. 1993; Hanawa et al. 1993; Matsumoto et al. 1994; Fiege \& Pudritz 2000a,b; Schleicher \& Stutz 2018; Reissl et al. 2018). Fiege \& Pudritz (2000a,b) studied the fragmentation length scale, stability, density profile, and mass per length of filamentary MCs, and, based on observational constraints, they suggest that many filamentary structures are likely wrapped by helical magnetic fields. Other studies of Orion-A, indirectly suggest a helical magnetic field morphology for this region (e.g., Johnstone \& Bally 1999; Matthews et al. 2001; Buckle et al. 2012; Contreras et al. 2013; Stutz \& Gould 2016; Hoq et al. 2017). For example, by using the Virial mass per length obtained by Fiege \& Pudritz (2000a) for a cylindrical filament threaded by a helical magnetic field, Buckle et al. (2012) showed that the integral shaped filament in Orion-A is too massive for thermal or turbulent support. Therefore, they suggest that the mass and morphology of the integral shaped filament (a small region within our defined Orion-A) is consistent with a Virial model of a filamentary structure threaded by a helical magnetic field.

Heiles (1997) proposed an alternative explanation, which associates the magnetic reversal in Orion-A to the Eridanus shock and its interaction with the dense MC. In this mechanism, the magnetic fields bend around the Orion $\mathrm{MC}$, as illustrated in Fig. 1. The ambient Galactic $B_{\|}$in this region is, in general, towards the observer, and interacts with the superbubble, generating the reversal (Heiles 1997). Heiles (1997) suggests that a reversal in this region can be observed even without the presence of an MC, however, the existence of an MC in this region makes this reversal sharper. This model is supported by the Planck observations considered in Soler et al. (2018), who mapped $\mathrm{B}_{\perp}$ lines in the Orion-Eridanus super-bubble, and suggested that, due to the large-scale shape of the magnetic field lines, $\mathrm{B}_{\perp}$ interacts with, and is influenced by, the Orion-Eridanus superbubble.

We refer to this morphology, regardless of how it is formed, as a bow morphology. This general morphology was recently investigated in a number of theoretical studies (Gómez et al. 2018; Inoue et al. 2018; Reissl et al. 2018), as well as observational studies (Liu et al. 2018), and has been also referred to as a U-shape or a pinched field.

Understanding the overall 3D magnetic field morphology, and consequently identifying which of these proposed magnetic configurations are responsible for the observed magnetic field reversals, can potentially help us answer questions pertaining to the role of magnetic fields in star formation. For example, helical fields can allow for more mass accumulation in the filamentary structure by stabilizing the cloud against self-gravity (Buckle et al. 2012). Additionally, since the magnetic fields
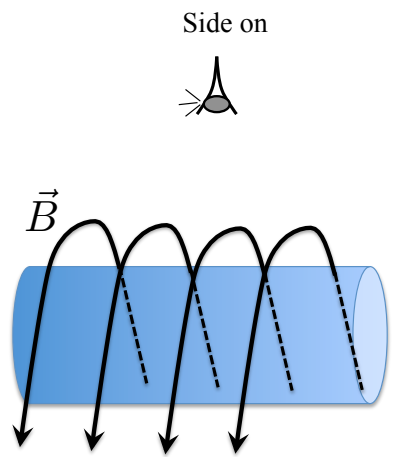

End on

$\rightarrow A$

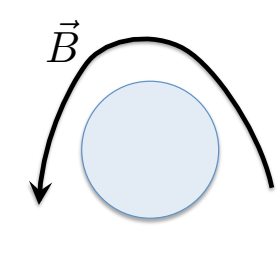

Fig. 1. Wrapping of magnetic fields lines around a cylindrical filamentary structure (blue circle), as seen by observers. This concept could provide an explanation for the observations seen in Orion-A, interacting with the Orion-Eridanus superbubble. The magnetic field line is represented by the curved black line.

must form closed loops (zero-divergence), determining the 3D magnetic field morphology in and around these filaments can provide information regarding the influence that the surrounding environment has on the formation and evolution of filamentary structure.

In this paper, we investigate the large-scale 3D magnetic field in Orion-A (the entire southern complex). For this purpose, we use PXXXV dust polarization data and the TPBK18 $\mathrm{B}_{\|}$ results. We discuss each of these datasets in Sect. 2, and describe our methodology to couple these two magnetic fields' results in Sect. 3. Finally, we discuss our results and interpretation. The goals of TPBK18 and this paper are to provide techniques to obtain the large-scale structure of magnetic fields around star forming MCs. The broad-perspective intent is to show what can be done with the existing data, as well as to set the stage for future, higher sensitivity RM catalogs, which will be obtained by next generation surveys and telescopes.

\section{Observations}

To investigate the 3D magnetic field we require both the line-ofsight and plane-of-sky components of magnetic fields. Below, we discuss the data we use for each of these components of the magnetic field in our study.

\subsection{Line-of-sight magnetic field component}

For the parallel component of the magnetic field $\left(\mathrm{B}_{\|}\right)$, we used the directions and magnitudes found in TPBK18. They calculate $\mathrm{B}_{\|}$using Taylor et al. (2009) rotation measure (RM) catalog, in combination with an electron column density estimated for each RM source from a chemical evolution code and Kainulainen et al. (2009) extinction maps. Additionally, they find $B_{\|}$reversal across two regions of California and Orion-A molecular clouds. TPBK18 explains in detail how to find the $\mathrm{B}_{\|}$strengths and their uncertainty values. These uncertainties are calculated for each data point separately, and are the result of the uncertainty propagation of all the required variables (input parameters) in determining $\mathrm{B}_{\|}$strength.

For this paper, we only considered the magnetic field around Orion-A, and used the data points that provide a solid direction for $B_{\|}$(either towards or away from us). Consequently, if a $B_{\|}$ value has an uncertainty bigger than the value of $B_{\|}$itself, we do not consider that data point in our analysis. That is, we use the $\mathrm{B}$ values with uncertainties $<100 \%$ so that the direction of 


\section{$B_{\text {LOS }}$ in OMC-A}

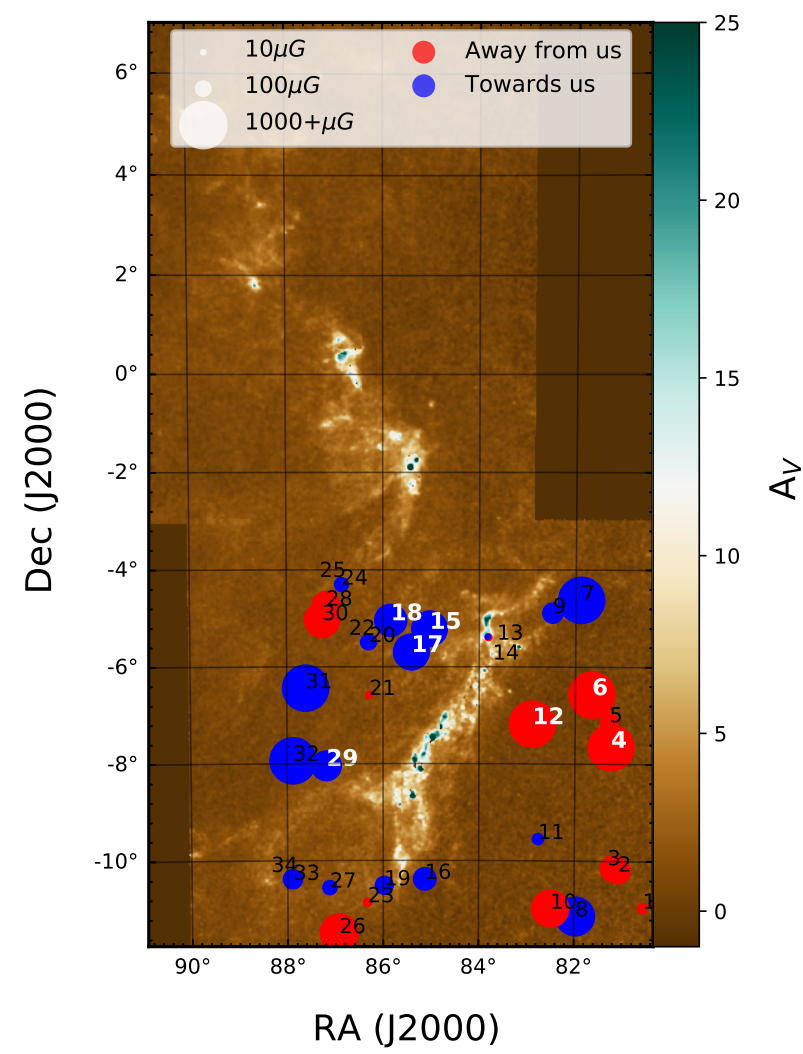

Fig. 2. $B_{\|}$in Orion-A. Blue (red) circles show magnetic fields toward (away from) us. The size of the circles indicates the magnitude of magnetic field. Sources numbered in bold white indicate the sources used for this study. Points 3, 4, 6, 7, 8, 12, 15, 17, 18, 19, 29, 31, 32, and 34 in this figure have errors less than $100 \%$. Points $4,6,12,15,17,18$, and 29 are considered in this study.

the field is well determined. For example, point 13 with $\mathrm{B}_{\|}=$ $-23 \pm 38 \mu \mathrm{G}$ (an uncertainty $>100 \%$ ) changes direction within its uncertainty range. We do not consider this point in our analysis, since its direction is highly uncertain. Figure 2 shows all the data points determined in TPBK18. Points 3, 4, 6, 7, 8, 12, 15, 17, $18,19,29,31,32$, and 34 in this figure have errors less than $100 \%$. Points $4,6,12,15,17,18$, and 29 , are considered in this study, since these are relatively close to the filamentary structure (filament) and are within the main axis length of the filament (see Sect. 3.1). In other words, these are the points with nearby $B_{\perp}$.

\subsection{Plane-of-sky magnetic field component}

For the plane-of-sky component $\left(\mathrm{B}_{\perp}\right)$, we used the PXXXV determination of the magnetic field orientation as projected on the plane of the sky. As explained in PXXXV, we found the magnetic field lines from the thermal emission of interstellar dust observed by Planck ${ }^{1}$ at $353 \mathrm{GHz}$ taken at an original resolution of $4^{\prime} .8$ and then smoothed with a $15^{\prime}$ FWHM Gaussian beam to guarantee signal-to-noise ratio $(\mathrm{S} / \mathrm{N})$ of at least three.

To find the average $B_{\perp}$ value in the Orion-A region, we used the Planck dust polarization data along with the method of Davis-Chandrasekhar-Fermi (DCF), plus the structure function (DCF+SF) described by Hildebrand et al. (2009). To obtain

1 https://www.esa.int/Our_Activities/Space_Science/ Planck the DCF components, we used the Planck $353 \mathrm{GHz}$ data for the angle dispersion and $\mathrm{CO}$ observations for the density and line-ofsight velocity dispersion. The value we obtained is $180 \pm 90 \mu \mathrm{G}$. This is a refined value tailored for Orion-A only, compared to what is mentioned in Table D.1 in PXXXV, which is for the entire Orion complex. Similar to $\mathrm{B}_{\|}$, the $\mathrm{B}_{\perp}$ uncertainty is as the result of uncertainty propagation of the input parameters (density and velocity dispersion) into determination of $\mathrm{B}_{\perp}$. Even though these values do not represent $B_{\perp}$ magnitude for each data point of TPBK18 Orion-A map, where there is $\mathrm{CO}$ emission, the $\mathrm{B}_{\perp}$ orientation at each point is determined from the Stokes parameters at that point.

\section{Methodology}

To investigate the validity of possible magnetic field morphologies, we modeled the different proposed configurations and compared the results of averaged magnetic fields along the LOS to those of observations. Below we discuss our methodology of combining the $\mathrm{B}_{\perp}$ and $\mathrm{B}_{\|}$results, constructing the models, and comparing the results of the models with the observations.

\subsection{Integrating the observations with models}

In order to compare the modeled values with the observations, we need to find $a B_{\perp}$ value and orientation that can be associated with the $\mathrm{B}_{\|}$at each point from TPBK18. We did this by finding the closest $\mathrm{PXXXV} \mathrm{B}_{\perp}$ to the $\mathrm{B}_{\|}$points. If no $\mathrm{B}_{\perp}$ value existed within $15^{\prime}$ (the size of the smoothed Planck polarization beam) of the $\mathrm{B}_{\|}$point, we discarded that $\mathrm{B}_{\|}$point from our analysis. This also means that the $\mathrm{B}_{\|}$data point is too far from the filament (points 3, 8, 19 in Fig. 2). Points 31, 32, 34 and 7 are also too far away from the filament axis. Therefore, we carried out this study with points numbered $4,6,12,15,17,18$, and 29 , as indicated by the white numbers in Fig. 2.

After finding the closest $\mathrm{B}_{\perp}$ point to our $\mathrm{B}_{\|}$point, we found the orientation of $\mathrm{B}_{\perp}$ by determining its polarization angle using

$\psi=\frac{1}{2} \arctan (-U, Q)$,

where $\psi$ is the polarization angle in the International Astronomical Union (IAU) convention, and $U$ and $Q$ are the Stokes parameters obtained from Planck observations of dust thermal radiation. We added a $\pi / 2$ to $\psi$ to account for the fact that the magnetic field orientation $(\phi)$ is orthogonal to the polarization angle. These angles were initially found with respect to the Galactic coordinate. To analyze the $3 \mathrm{D}$ geometry of the magnetic field with respect to the filament itself, we converted (rotated) these angles to the frame of reference of the filament itself.

We set the filament frame of reference so that the $x$-axis was radial to the filament, and in the plane of the sky (i.e., the short axis of the filament in the plane of the sky from east to west), the $y$-axis was parallel to the long axis of the filament in the plane of the sky, and the $z$-axis was radial, towards the observer, as illustrated in Fig. 3. We were then able to write the magnetic field vector at each point in this frame of reference as $\mathbf{B}=\left(B_{x}, B_{y}, B_{z}\right)$. Compared to the Galactic system, this new frame of reference is rotated by $\xi$, where $\xi$ is the angle the filament's minor axis makes with Galactic longitude axis in the decreasing direction, meaning, the orientation of the $x$-axis of the filament with respect to east-west direction of the map measured in a clockwise direction.

In this system, $\left|B_{x}\right|=\left|B_{\text {Planck }} \sin (\xi+\phi)\right|$ and $\left|B_{y}\right|=$ $\left|B_{\text {Planck }} \cos (\xi+\phi)\right|$, where $\phi$ is the magnetic angle in the plane 


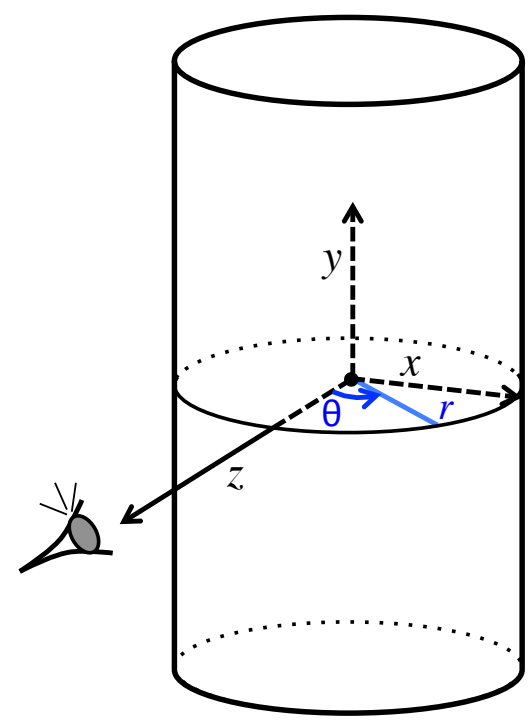

Fig. 3. Geometry and coordinates for a filament. The $x$-axis is radial to the filament, and in the plane of the sky, $y$-axis is parallel to the long axis of the filament, and $z$-axis is radial, towards the observer.

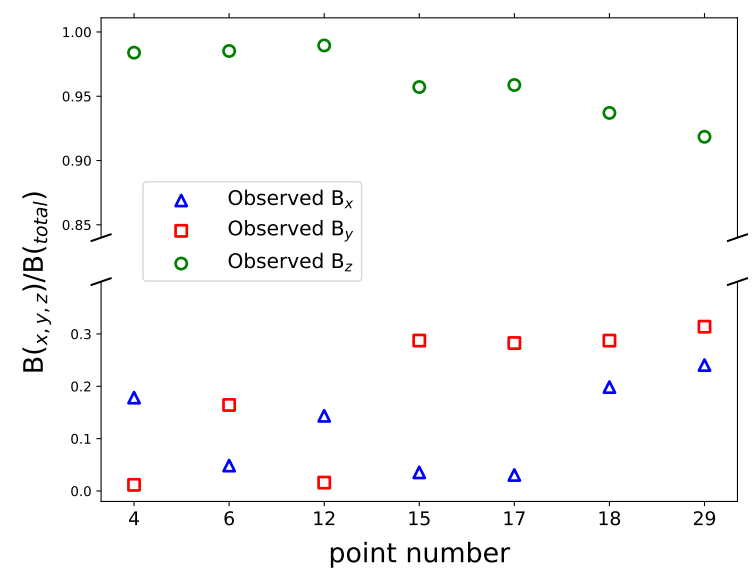

Fig. 4. Observed $B_{x}, B_{y}$, and $B_{z}$ using frame system depicted in Fig. 3 , as well as $\mathrm{B}_{\perp}$ and $\mathrm{B}_{\|}$values obtained by PXXXV and TPBK18, respectively. $Y$-axis shows the ratio of each component of magnetic field to the total value, and $x$-axis refers to the numbered data points labeled in Fig. 2.

of the sky and $B_{\text {Planck }}=180 \pm 90 \mu \mathrm{G}$. The equations are cast as absolute values, because we do not yet have any information regarding the positive or negative signs of $B_{x}$ and $B_{y}$ that provide the direction of $\mathrm{B}_{\perp}$. While the orientation of $\mathrm{B}_{\perp}$ can be identified from the Stokes parameters, the actual direction (+ or - along that orientation) cannot be determined. For the $z$ axis, $B_{z}=\mathrm{B}_{\|}$and does include the positive and negative signs of $\mathrm{B}_{\|}$.

Figure 4 shows the resultant magnetic fields in the Cartesian frame illustrated in Fig. 3. In this figure, we took the ratio of the observed magnetic field components (i.e., $B_{x}, B_{y}, B_{z}$ ) to the total estimated observed magnetic field strength $\left(B_{\text {total }}=\right.$ $\left.\sqrt{B_{x}^{2}+B_{y}^{2}+B_{z}^{2}}\right)$. Considering the ratios helps us reduce the number of free parameters in the models. To distinguish between morphologies, we compared these observed magnetic field strengths to those predicted by our three models as described in sections below.

\subsection{Modeling a toroidal and a helical 3D magnetic field}

We model a filament with a simple toroidal or a helical magnetic field using the following set of equations:

$$
\begin{aligned}
& B_{x}=\frac{B_{0} \mathrm{R} \cos (\theta)}{r^{\alpha}}=\frac{B_{0} \mathrm{R} z}{\left(x^{2}+z^{2}\right)^{\frac{\alpha+1}{2}}}, \\
& B_{y}=\frac{B_{1} \mathrm{R}}{r^{\alpha}}=\frac{B_{1} \mathrm{R}}{\left(x^{2}+z^{2}\right)^{\frac{\alpha}{2}}}, \\
& B_{z}=\frac{-B_{0} \mathrm{R} \sin (\theta)}{r^{\alpha}}=\frac{-B_{0} \mathrm{R} x}{\left(x^{2}+z^{2}\right)^{\frac{\alpha+1}{2}}},
\end{aligned}
$$

where the $x, y$, and $z$ directions follow the convention set in Sect. 3.1 and Fig. 3. $R$ is a scaling factor with units of [length] ${ }^{\alpha}$ and $r=\sqrt{x^{2}+z^{2}}-$ the radial distance from the filament midplane in the $X-Z$ plane (i.e., perpendicular to the long axis). $B_{0}$ and $B_{1}$ carry information about the strength of the toroidal and poloidal components of magnetic field, respectively. The parameter $\alpha$ reflects the rate at which the magnetic field strength decreases with distance from the filament.

To have a purely toroidal magnetic field, or a helix with a small pitch angle, $B_{1}$ needs to be either zero or very small compared to $B_{0}$. In these equations, $\frac{x}{\left(x^{2}+z^{2}\right)^{1 / 2}}$ and $\frac{z}{\left(x^{2}+z^{2}\right)^{1 / 2}}$ replace $\sin \theta$ and $\cos \theta$.

Since a helical field threading a filament must be caused (produced) by the filament itself, we assume its strength drops with distance from the filament's main axis. The scaling factor $R$, therefore, arises from the relation between the strength of the magnetic field and its distance from the filament. This ensures that the units of both sides of these equations are identical (i.e., units of magnetic field strength).

To understand the rationale behind the magnetic strength decreasing with $1 / \mathrm{r}^{\alpha}$, we consider the following: the relation between the magnetic field and MC gas density; the observed density profile for the Orion-A filament; the necessity to keep the magnetic field divergence free.

The relationship between the magnetic field strength and the gas density has been investigated both theoretically and observationally. Theoretically, Tritsis et al. (2015) found that $B(\rho) \propto \rho^{1 / 2}$ is preferred, where $B(\rho)$ is the magnetic field as a function of the mass volume density and $\rho$ is the mass volume density. Observationally, Crutcher et al. (2010) propose a ratio of $B(\rho) \propto \rho^{2 / 3}$ when the magnetic energy is small compared to the effects of gravity. In our models, we consider both $B(\rho) \propto \rho^{1 / 2}$ and $B(\rho) \propto \rho^{2 / 3}$.

The density profile of the Orion-A filament has also been studied both observationally and theoretically and is proposed to have a Plummer-like form (e.g. Salji et al. 2015). The standard Plummer profile is described by:

$\rho(r)=\frac{\rho_{\mathrm{c}}}{\left[1+\left(r / r_{\text {flat }}\right)^{2}\right]^{p / 2}}$,

where $\rho_{\mathrm{c}}$ is the central density of the filament, $r$ is the distance (radius) from the filament axis, and $r_{\text {flat }}$ is the characteristic radius defining the central region, where the density profile flattens. The exponent $p$ is observationally determined and sets the density drop-off rate. However, we use the more recently defined density profile suggested by Stutz \& Gould (2016, see their Eq. (5)), where they suggest that $\rho(r) \propto r^{-13 / 8}$. This density profile not only fits the observations, it also keeps our magnetic field divergence-free (i.e., $\frac{\partial B_{x}}{\partial x}+\frac{\partial B_{y}}{\partial y}+\frac{\partial B_{z}}{\partial z}=0$ in Eq. (2)). 
We extended this density profile to the larger Orion-A filamentary structure. Combining $\mathrm{B}(\rho)$ with $\rho(r)$, we obtained a relation for $\mathrm{B}(r)$ in which the magnetic field strength decreases as $1 / \mathrm{r}^{\alpha}$, where $\alpha$ can be either $13 / 16$ or $13 / 12$ depending on whether we used the Tritsis et al. (2015) or the Crutcher et al. (2010) relations.

\subsection{Modeling a bow magnetic field morphology}

The simplest divergence-free magnetic field relation one can use to describe a bow morphology is:

$B_{x}=B_{1}$,

$B_{y}=B_{2}$,

$B_{z}=B_{0} \frac{x}{\mathrm{R}^{\prime}}$

where $B_{0}, B_{1}$, and $B_{2}$ are free parameters with positive values which provide the strength of magnetic field. $\mathrm{R}^{\prime}$ is a free parameter with the unit of length to ensure that the relation for the $z$-component of magnetic field in Eq. (4) at both sides have the same units (see Sect. 4.3 for a range of values explored for these free parameters). $B_{x}$ and $B_{y}$ remain constant and in the direction of the ambient magnetic field, whereas $B_{z}$ varies with $x$. Since the value of the $x$-coordinate varies from positive to negative across the filament, this implies that $B_{z}$ reverses direction from one side of the filament to the other.

It is important to note that these equations represent single magnetic field lines. If we follow one field line as it wraps around a filament, we see that the $z$-component (towards the observer) of the field is zero directly in front of, and behind, the filament. As we increase the radial distance from the filament ( $x$-direction), the $z$-component becomes larger. Thus, this relation of $B_{z}$ with $x$ represents the wrapping of the field lines around the filament, as well as the reversal.

Based on the model proposed by Heiles (1997), we assumed that in the bow morphology, the magnetic field components $\left(B_{x}, B_{y}, B_{z}\right)$ represent field lines that do not penetrate the dense filament but, instead, represent the ambient magnetic field. Since they are ambient field lines, we do not expect a change of B-field strength as a function of distance from the filament's long-axis. Therefore, we made the following assumptions: in the above set of equations for the bow model, the overall magnetic field strength does not decrease with distance; $B_{1}=B_{2}$ represents the ambient magnetic field. Thus, we only explore different ratios of $B_{0}$ and $B_{1}$ (see Sect. 5).

If we forgo the $\nabla \cdot \mathbf{B}=0$ requirement, we can use the equations provided by Reissl et al. (2018), which model the bow morphology in a slightly different manner:

$B_{x}=\frac{B_{0}(x, z)}{1+U^{2}(x, z)}$,

$B_{y}=0$,

$B_{z}=\frac{B_{0}(x, z) U(x, z)}{1+U^{2}(x, z)}$

with

$U(x, z)=-5 x(2-z)^{2} e^{-8 x^{2}}$,

$B_{0}(x, z)=\frac{B_{0}}{\left(1+\left(r / r_{\text {flat }}\right)^{2}\right)^{-0.6 \beta}} ; \quad r=\sqrt{x^{2}+z^{2}}$,

where $r_{\text {flat }}$ is the characteristic radius of the Plummer-like density profile (i.e., where the profile becomes flat close to the center of the filament). The parameter $\beta$ controls the slope of the density drop-off in the outer regions. Reissl et al. (2018) used $\beta=1.6$, which is the average value of $\beta$ from the Arzoumanian et al. (2011) density profile. $B_{0}$ controls the total strength of the magnetic field.

\subsection{Comparing observed $3 D$ magnetic field strengths with models}

Since, along any given LOS, we see a superposition of many different field lines, the observed magnetic field components $\left(B_{x}, B_{y}, B_{z}\right)$ are averages over the LOS. To account for this in our models, we find the average magnetic field components along the LOS for each $x, y$, and $z$ component of the field using the following integral:

$\left\langle B_{i}\right\rangle(x / L)=\frac{\int_{-\frac{L}{2}}^{+\frac{L}{2}} B_{i}(x, z) \mathrm{d} z}{L}$,

where $L$ is the integration length along the LOS, and $i$ indicates $x, y$, or $z$ components. Therefore, $\left\langle B_{i}\right\rangle(x / L)$ is the average value of the $x, y$, or $z$ component of the magnetic field at a radial distance of $x$ from the filament long axis (the $y$-axis), and averaged over the line-of-sight distance of $L . B_{i}(x, z)$ represents the magnetic field components from each model in Eqs. (2), (4), and (5). This calculation is performed for each of the models (toroidal, helical, and bow) separately. Thus, Eq. (7) provides $\left\langle B_{x}\right\rangle,\left\langle B_{y}\right\rangle$, and $\left\langle B_{z}\right\rangle$ as a function of $x$ (perpendicular distance to the filament axis) and $L$ (integration path). We explored these averages as a function of $x / L$ to avoid the need to convert $x$ and $L$ into real distances and reduce the number of free parameters in the analysis. We set this integration distance $(L)$ to be half of the distance of the point 17 from the filament axis, because we assume this distance represents the MC's thickness along the LOS. One should note that finding the observed $\mathrm{B}_{\|}$values do not require the actual integration distance, since TPBK18 use the column density values instead of the volume densities. Information about the lineof-sight integration distance is already embedded in the column densities.

To compare the models with the observed values, we determined the perpendicular distance $(x)$ of each of our selected points in Fig. 2 from the filament axis. Additionally, we found the ratio of each $x, y$, and $z$ component to the total (averaged along the LOS) magnetic field - both in the models and in the data, i.e., $\left(\frac{\left\langle B_{x}\right\rangle}{\left\langle B_{\text {total }}\right\rangle}, \frac{\left\langle B_{y}\right\rangle}{\left\langle B_{\text {total }}\right\rangle}, \frac{\left\langle B_{z}\right\rangle}{\left\langle B_{\text {total }}\right\rangle}\right)$, where $B_{\text {total }}=\sqrt{\left\langle B_{x}\right\rangle^{2}+\left\langle B_{y}\right\rangle^{2}+\left\langle B_{z}\right\rangle^{2}}$. These represent the ratios that should be observed, ideally without experimental uncertainties and systematic observing biases. We then compared the above-mentioned ratios between the observed and modeled magnetic field components in order to determine which model is most consistent with the observed data. We performed this analysis in Sect. 4.

To compare the data with the models, we employed a $\chi^{2}$ approach, using the following relation for each component of the magnetic field.

$\chi_{i}^{2}=\sum_{j=1}^{N} \frac{\left(\text { observed } \frac{\left\langle B_{i}\right\rangle}{\left\langle B_{\mathrm{tot}}\right\rangle}-\operatorname{modeled} \frac{\left\langle B_{i}\right\rangle}{\left\langle B_{\mathrm{tot}}\right\rangle}\right)_{j}^{2}}{\delta_{j}^{2}}$,

$\chi_{\text {tot }}^{2}=\chi_{x}^{2}+\chi_{y}^{2}+\chi_{z}^{2}$,

where $i$ represents the $x, y$, or $z$ component of the magnetic field. $\mathrm{N}$ is the number of observational points in the study (in this paper $N=7$ ), and $\chi_{\text {tot }}^{2}$ is the total $\chi^{2}$. "Observed $\frac{\left\langle B_{i}\right\rangle}{\left\langle B_{\mathrm{tot}}\right\rangle}$ " is the observed 
ratio of each component of magnetic field to the total magnetic field as plotted in Fig. 4. "Modeled $\frac{\left\langle B_{i}\right\rangle}{\left\langle B_{\text {tot }}\right\rangle}$ " is the LOS average of each model as explained in Sect. 3.4. $\delta$ represents the uncertainties of the magnetic field strengths determined in $\mathrm{B}_{\|}$and $\mathrm{B}_{\perp}$ combined. This $\delta$ is the unit-less relational uncertainty for the ratios as follows:

$\delta=\sqrt{\left(\frac{\delta B_{\|}}{B_{\|}}\right)^{2}+\left(\frac{\delta B_{\perp}}{B_{\perp}}\right)^{2}}$,

where $\delta B_{\|}$is the uncertainty of $\mathrm{B}_{\|}$and $\delta B_{\perp}$ is the uncertainty of $\mathrm{B}_{\perp}$. For example, for point number 29 in Fig. 2, with $\mathrm{B}_{\|}=-418 \pm 308 \mu \mathrm{G}$ and $\mathrm{B}_{\perp}=180 \pm 90 \mu \mathrm{G}, \delta$ is calculated as follows:

$\delta=\sqrt{\left(\frac{308}{418}\right)^{2}+\left(\frac{90}{180}\right)^{2}}=0.89$.

\section{Results of modeling}

Figure 4 demonstrates the ratio of the observed magnetic field components (i.e., $B_{x}, B_{y}, B_{z}$ ) to the total estimated observed magnetic field strength $\left(B_{\text {total }}=\sqrt{B_{x}^{2}+B_{y}^{2}+B_{z}^{2}}\right)$ in the filament's Cartesian frame shown in Fig. 3. To determine which of our three models best fits the data, we compare the predicted magnetic field strengths for each of the models to these observed values.

\subsection{The toroidal morphology}

We investigated the toroidal model using Eq. (2) and setting $B_{1}=$ 0 (i.e., a helix with zero pitch angle). By considering the ratios of each component of the magnetic field to the total averaged magnetic field value, the free parameters $R$ and $B_{0}$ cancel out, and do not contribute to the final results.

The top row of Fig. 5 shows the results of the toroidal model for the two different assumptions of the relationship between magnetic field strength and density: alpha $=13 / 16$ (left) and 13/12 (right). The symbols represent the observed values, and the lines depict the model results as described in Sect. 3.4. The blue, red, and green colors illustrate the $x, y$, and $z$ components, respectively. Both alpha values produce identical model results, which indicates that the decrease in magnetic field strength with distance does not play a noticeable role.

The top row of Fig. 5 suggests that while the toroidal model seems to provide a good match to the z-component of the magnetic field, the modeled $x$ and $y$ components do not. The corresponding $\chi^{2}$ values for each component are: $\chi_{x}^{2}=0.109$, $\chi_{y}^{2}=0.778, \chi_{z}^{2}=0.020$, and $\chi_{\text {tot }}^{2}=0.908$ for both $\alpha=13 / 16$ and $13 / 12$.

\subsection{The helical morphology}

The pitch angle of a helix can be parameterized by the ratio of $B_{1} / B_{0}$ in Eq. (2). Increasing this ratio increases the pitch angle. After taking the LOS averages (see Sect. 3.4), $B_{1}$ and $B_{0}$ do not cancel out, but instead remain as free parameters. Thus, to study a helical model, we used Eq. (2) and varied the ratio of $B_{1} / B_{0}$, from 0.0 to 1.49 in step sizes of 0.01 . For each value of $B_{1} / B_{0}$, we calculated the corresponding $\chi^{2}$ value. The minimum $\chi^{2}$ is calculated as the sum of the $\chi^{2}$ for all the components (i.e., $\chi_{\text {tot }}^{2}$ ).
We also paid particular attention to the $y$-component, since it sets the toroidal model apart from the helical one. The lowest $\chi^{2}$ occurs for $B_{1} / B_{0}=0.21$ (both with $\alpha=13 / 16$ and 13/12), with $\chi_{x}^{2}=0.109, \chi_{y}^{2}=0.193, \chi_{z}^{2}=0.008, \chi_{\text {tot }}^{2}=0.310$. The two bottom rows of Fig. 5 represent the modeled helical morphology for $\alpha=13 / 16$ (left) and 13/12 (right). The middle, and the bottom rows show $B_{1} / B_{0}$ value of 0.21 (the best $\chi^{2}$ fit result) and 0.5 , respectively.

\subsection{The bow morphology}

To model the bow magnetic morphology, we used Eq. (4), and find the ratio of each magnetic field component to the total magnetic field (as explained in Sect. 3.4). Since the free parameters $B_{1}, B_{0}$, and $\mathrm{R}^{\prime}$ do not cancel out, we explored different values of $B_{0} / B_{1}$ and $\mathrm{R}^{\prime}$. To explore these free parameters, we first picked arbitrary values that result in a best visual fit with the data. For $\mathrm{R}^{\prime}$, this initial value is equal to the integration distance, meaning, half of the perpendicular distance of point 17 from the filament axis. We then explored the influence of larger and smaller values of these free parameters in a quantitative manner. In addition, in Eq. (4), we assume $B_{2}=B_{1}$, so that the $\mathrm{x}$ and $\mathrm{y}$ components of the magnetic field are equal, and then explore a range to find the lowest $\chi^{2}$ results. Since the observed $B_{\|}$values are higher than the Planck $\mathrm{B}_{\perp}$ average strength, we started from $B_{1} / B_{0}=0.5$ and calculate the $\chi^{2}$ for various $\mathrm{R}^{\prime}$ values up to $B_{1} / B_{0}=15$, with step size of 0.5 . We took this range of parameters because we found that they fit the data better visually. In this range of $B_{1} / B_{0}$, we explored the $\mathrm{R}^{\prime}$ values from 0.3 of the integration distance $(0.3 \mathrm{~L})$ up to $3.2 \mathrm{~L}$, with step-size of 0.1 . Figure 6 shows the results for this bow model for two sets of free parameters, the left panel represents a set of parameters that produce the smallest $\chi^{2}$ result. The best-fit model is for $B_{0} / B_{1}=3.5$ and $\mathrm{R}^{\prime}=1.3 \mathrm{~L}$ with $\chi_{x}^{2}=0.158, \chi_{y}^{2}=0.181, \chi_{z}^{2}=0.007, \chi_{\text {tot }}^{2}=0.346$.

To explore the bow model further, we consider Eq. (5), as described in Sect. 3.3. For this purpose, we modified the equations slightly to represent a more general form and to be compatible with our filament setup (i.e., to account for the reversal in the scales observed in TPBK18 and to make sure the units in the right and left hand sides of Eq. (5) are the same $[\mu \mathrm{G}])$. We set $U(x, z)$ in Eq. (6) as:

$U(x, z)=b_{\mathrm{R}} x\left(c_{\mathrm{R}}-z\right)^{2} \exp \left(a_{\mathrm{R}} x^{2}\right)$,

where $a_{\mathrm{R}}, b_{\mathrm{R}}$, and $c_{\mathrm{R}}$ are free parameters. These parameters help the observed and modeled results to be close to each other visually.

To explore a range of free parameters, we set $a_{\mathrm{R}}, b_{\mathrm{R}}$, and $c_{\mathrm{R}}$ in form of $\frac{-8}{(a \mathrm{~L})^{2}}, \frac{-5}{(b \mathrm{~L})^{2}}$, and $c_{\mathrm{R}}=c \mathrm{~L}$, where $a, b$, and $c$ are scalar values. In order to reduce the number of free parameters, we take the parameters as a function of $\mathrm{L}$. These forms result in a unit of $\mu \mathrm{G}$ in Eq. (5). We subsequently explore different $\mathrm{a}, \mathrm{b}$, and $\mathrm{c}$ values where $5 \leq a \leq 17$ with step-sizes of $0.5,0.2 \leq b \leq 3$ with step-size of 0.2 , and $1 \leq c \leq 15$ with step-sizes of 1 . Figure 7 shows the results for $\left[a_{\mathrm{R}}=\frac{-8}{(9.5 \mathrm{~L})^{2}}, b_{\mathrm{R}}=\frac{-5}{(2.2 \mathrm{~L})^{2}}\right.$, and $\left.c_{\mathrm{R}}=10 \mathrm{~L}\right]$ and for $\left[a_{\mathrm{R}}=\frac{-8}{(8 \mathrm{~L})^{2}}, b_{\mathrm{R}}=\frac{-5}{\mathrm{~L}^{2}}\right.$, and $\left.c_{\mathrm{R}}=3 \mathrm{~L}\right]$. We noticed that different values of $r_{\text {flat }}$ did not generate any noticeable differences in final results, since we consider the ratios.

Since the $\mathrm{B}_{y}$ component is zero in this version of bow model, $\chi_{y}^{2}$ is constant and determined to be 0.778 . This is lower than that of the toroidal model, but higher than those of both the helical and bow models from Eq. (4). We found that a few sets of parameters with $a=9.5$ result in the lowest $\chi^{2}$ with $c$ in a range of 9-13 
M. Tahani et al.: Bow magnetic field morphology?
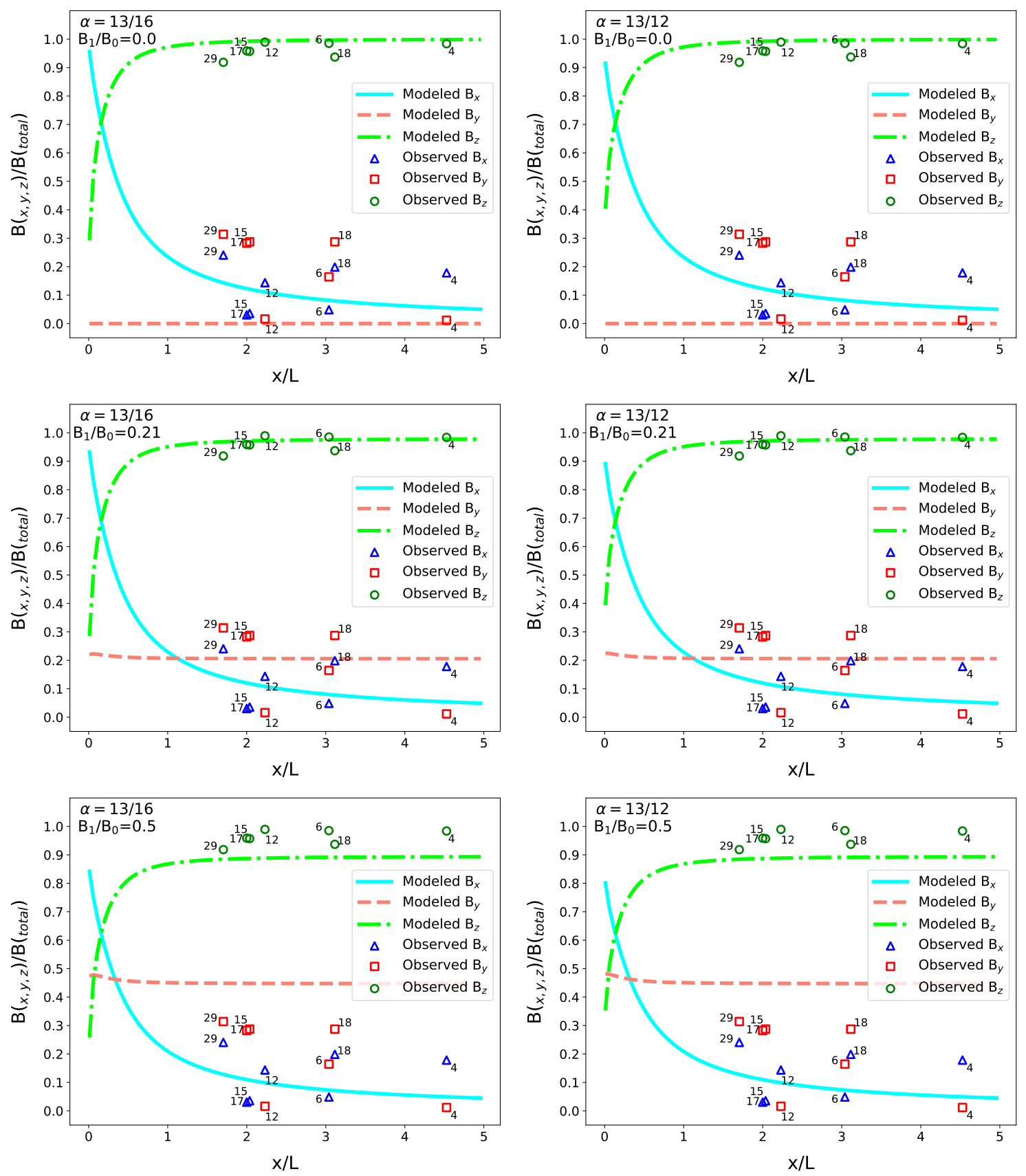

Fig. 5. Best fits for modeled toroidal and helical fields. Colored lines represent the modeled $x, y$, and $z$ components of the magnetic field. The colored symbols represent the $x, y$, and $z$ components of the observed magnetic field. On $x$-axis, $X$ is the perpendicular distance from the filament's long axis, and $L$ is the LOS integration distance. The two $\alpha$ values are the parameters explained in Sect. 3.2. The different $B_{1} / B_{0}$ ratios arise from Eq. (2) and set the level of helicity, with $B_{1} / B_{0}=0$ indicating a purely toroidal field.

and $\mathrm{b}$ in range of 2.0-2.8. For example $a=9.5, b=2.2$, and $c=$ 10 (i.e., $a_{\mathrm{R}}=\frac{-8}{(9.5 \mathrm{~L})^{2}}, b_{\mathrm{R}}=\frac{-5}{(2.2 \mathrm{~L})^{2}}$, and $\left.c_{\mathrm{R}}=10 \mathrm{~L}\right)$ or $a=9.5, b=$ 2.4 , and $c=11$ produce $\chi^{2}$ result of $\chi_{x}^{2}=0.073, \chi_{y}^{2}=0.778$, $\chi_{z}^{2}=0.021, \chi_{\text {tot }}^{2}=0.872$. Given the small step-sizes having a range for the smallest $\chi^{2}$ is expected. For this bow model, we picked the set of parameters, which, when we considered more significant digits of $\chi^{2}$, result in the smallest $\chi^{2}$. That is with $a_{\mathrm{R}}=\frac{-8}{(9.5 \mathrm{~L})^{2}}, b_{\mathrm{R}}=\frac{-5}{(2.2 \mathrm{~L})^{2}}$, and $c_{\mathrm{R}}=10 \mathrm{~L}$.

Finally, it should be noted that in Eqs. (5) and (6), the density, which is used to relate the magnetic field strength to distance from the filament in $B_{0}(x, z)$, is that of a Plummer-like profile. An alternative approach is to use the earlier proposed relation between the magnetic strength and $r$ (see Sect. 3.2) of

$B_{0}(x, z)=\frac{B_{0}}{\left(x^{2}+z^{2}\right)^{\frac{-\alpha}{2}}}$.

However, even with this density profile, Eq. (5) is not divergencefree for this bow model. We found that using the two different density profiles does not provide noticeably different results.

Finally, we note that these results are based on magnetic field values that have large uncertainties, and possibly have systematic biases. To alleviate the dependency of our interpretation of the $3 \mathrm{D}$ magnetic field morphology on sole values of $\mathrm{B}_{\perp}$ and $\mathrm{B}_{\|}$, we carry out a Monte Carlo analysis in Sect. 5. 

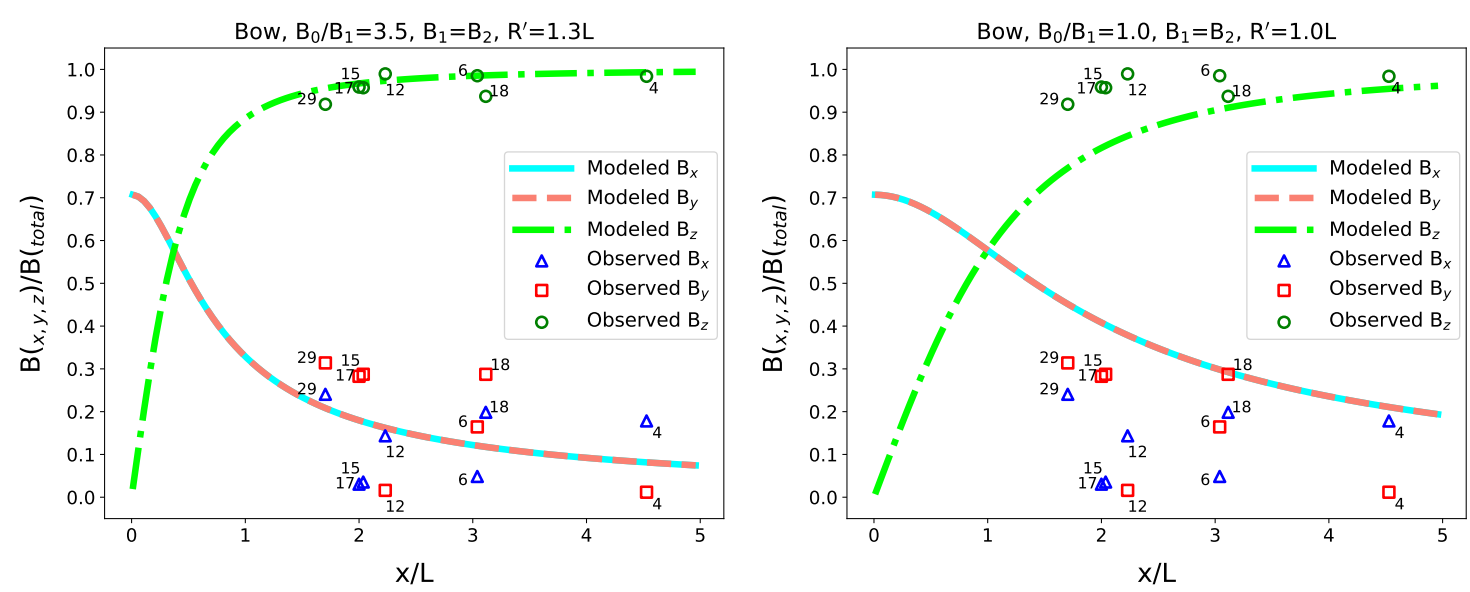

Fig. 6. Bow magnetic morphology from Eq. (4). The magnetic fields are averaged along the LOS. Colored lines represent the modeled $x, y$, and $z$ components of the magnetic field. The colored symbols represent the $x, y$, and $z$ components of the observed magnetic field. On the $x$-axis, $X$ is the perpendicular distance from the filament's long axis, and $L$ is the LOS integration distance. The parameters show different $B_{1} / B_{0}$ ratios from Eq. (4). Left panel shows best fit for Eq. (4).
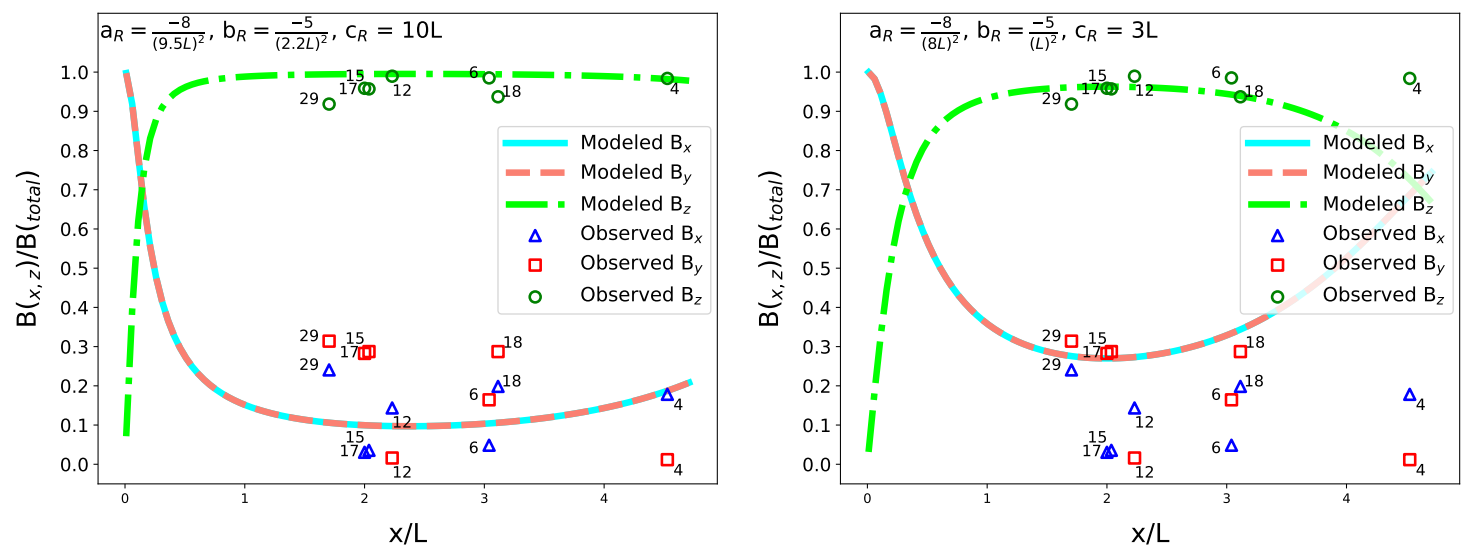

Fig. 7. Bow magnetic morphology from Eq. (5). The magnetic fields are averaged along the LOS. Colored lines represent the modeled $x, y$, and $z$ components of the magnetic field. The colored symbols represent the $x, y$, and $z$ components of the observed magnetic field. On $x$-axis, $X$ is the perpendicular distance from the filament's long axis, and L is LOS integration distance. Left panel shows best fit for Eq. (5).

\section{Discussion}

In this section, we explore the results further and propose the most likely magnetic field morphology for Orion-A. We then consider effects that may have biased our results.

\subsection{A Monte Carlo analysis}

PXXXV provide a magnetic field value $\left(\mathrm{B}_{\perp}\right)$ that is averaged over the entire Orion-A region, whereas TPBK18 provide the $\mathrm{B}_{\|}$ field values for very specific points with observed RMs. Thus, applying this single value of $\mathrm{B}_{\perp}$ to every $\mathrm{RM}$ position comes with significant uncertainties. In addition, the values of $B_{\|}$from TPBK18 come with their own, inherently large, uncertainties. To account for these uncertainties, we performed a Monte-Carlo error analysis to compare the models with the observed magnetic field components. In this approach, we randomly altered the $\mathrm{B}_{\|}$and $\mathrm{B}_{\perp}$ strengths of each single data point separately within their uncertainty ranges, and, for each iteration, compared the models with the data. More specifically we performed the following steps: (1) randomly changed $\mathrm{B}_{\|}$for each data point within its uncertainty range (from TPBK18); (2) randomly changed the strength of Planck magnetic field $\left(\mathrm{B}_{\perp}\right)$ for each data point separately within its uncertainty range $( \pm 90 \mu \mathrm{G})$; (3) calculated the "new" $B_{x}, B_{y}$, and $B_{z}$ values, using the "new" $\mathrm{B}_{\perp}$ and $\mathrm{B}_{\|}$values for each data point; (4) found the differences $(\varsigma)$ between the "new" $B_{x}, B_{y}$, and $B_{z}$ values and the best toroidal, helical, and bow models found in Sect. 4 using the equations:

$\boldsymbol{\zeta}_{x}=\sum_{i=1}^{N}\left(\left|B_{x, \text { modeled }}\right|-\left|B_{x, \text { observed }}\right|\right)_{i}^{2}$,

$\varsigma_{y}=\sum_{i=1}^{N}\left(\left|B_{y, \text { modeled }}\right|-\left|B_{y, \text { observed }}\right|\right)_{i}^{2}$,

$\boldsymbol{S}_{z}=\sum_{i=1}^{N}\left(\left|B_{z, \text { modeled }}\right|-\left|B_{z, \text { observed }}\right|\right)_{i}^{2}$,

$\varsigma_{\text {total }}=\varsigma_{x}+\varsigma_{y}+\varsigma_{z}$,

where $N$ is the number of data points $(N=7)$. We then found which of our best toroidal, helical, or bow models had the lowest $\zeta_{\text {total }}$ value when compared against the "new" (i.e., randomly altered) data. This Monte Carlo analysis was done 50000 times. Our bow model had the smallest difference (i.e., the bow model best fits the data) 34177 times. The helical model had the smallest difference 15823 times. In no cases did the toroidal model have the smallest difference. 


\subsection{Systematic biases}

Since $\mathrm{B}_{\perp}$ and $\mathrm{B}_{\|}$are derived in completely different ways (using Faraday rotation for $B_{\|}$and dust polarization for $B_{\perp}$ ), we probed the effects of potential systematic biases between the results of the two methods. Additionally, it should be noted that our values of $B_{\|}$are generally larger than the $B_{\perp}$ value and the $B_{\perp}$ value is an average for the region. Therefore, our $B_{\perp}$ could be systematically lower than $B_{\|}$. To investigate potential systematic biases, we explored a range of factors multiplied by our $\mathrm{B}_{\|}$ and $\mathrm{B}_{\perp}$ separately, and repeated a process similar to that presented in Sect. 5.1. In more detail, we followed the following steps: (1) multiplied all of our $B_{\|}$values by a randomly generated factor between 0.5 and five (same factor for all of the $\mathrm{B}_{\|}$values); (2) multiplied the $\mathrm{B}_{\perp}$ value by a randomly generated factor between one and five; (3) calculated the "new" $B_{x}$, $B_{y}$, and $B_{z}$ values, using the "new" $\mathrm{B}_{\perp}$ and $\mathrm{B}_{\|}$values for each data point; (4) found the differences $(\varsigma)$ between the "new" $B_{x}$, $B_{y}$, and $B_{z}$ values and the best toroidal, helical, or bow models found in Sect. 4, using Eq. (13). We then found which of our best toroidal, helical, or bow models had the lowest $\varsigma_{\text {total }}$ value when compared against the "new" data. This analysis was done 50000 times. Our bow model had the smallest difference (i.e., the bow model best fits the data) for 25002 times. The bow shape in form of Eq. (11) and the helical model had the smallest difference for 5195 and 19803 times, respectively. Therefore, considering this range of systematic biases, the bow model seems to be a better fit.

\subsection{Selecting the best magnetic field morphology}

To compare the toroidal and helical models, we only need to compare the $\chi^{2}$ values of the two models with each other. This is because these two models are a single model with different parameters (one with $B_{1} / B_{0}=0$ and the other with $B_{1} / B_{0}=$ 0.21 ). Therefore, since $\chi_{\text {tot }}^{2}=0.310$ for the helical model and 0.908 for the toroidal model, we suggest a helical morphology fits the data better. Furthermore, since it is the $y$-component that sets the toroidal and helical models apart, visually comparing the $y$-component in the top panel of Fig. 5 with the middle-left panel illustrates that a helical model (with $B_{1} / B_{0}=0.21$ ) is a better fit to the data. This is borne out in the mathematical analysis, which shows that $\chi_{y}^{2}=0.193$ for the helical model and 0.778 for the toroidal model.

To compare the bow morphology to the helical or toroidal one, we first compare the left panel of Fig. 6 and the left panel of Fig. 7 with the helical and toroidal models in Fig. 5. From these figures, it is easy to see that the bow models fit the data better than a toroidal model. However, it is difficult to see if either of the bow models fit the data better than the helical model. Thus, a more robust comparison is required.

Since the bow and helical (or toroidal) morphologies are represented by two very different models, one cannot simply compare their $\chi^{2}$ values. Thus, to compare the bow morphology to the toroidal or helical one, we compare their probability values (i.e., $p$-values). $P$-values allow one to compare different models by providing the probability for a statistically relevant model. The probability value provides the likelihood that a hypotheses (toroidal, helical, or bow) is true (Sivia \& Skilling 2006). In statistical analysis, $p$-values are normally used to reject a null hypothesis. If we assume that they represent a Gaussian distribution, it is a measure to understand whether the result from the hypothesis is closer to the peak of the distribution or its tail. A model or hypothesis can be ruled out if the $p$-value is lower than a threshold, typically 0.05 .

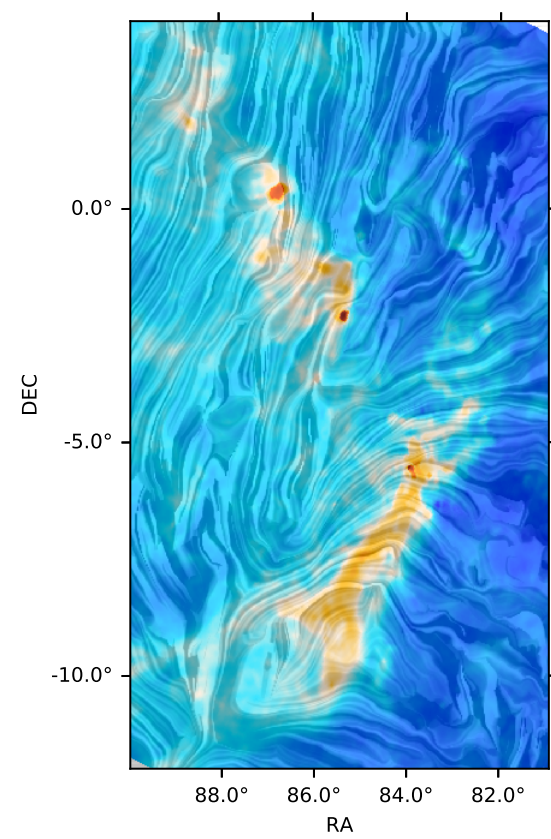

Fig. 8. Column density and magnetic field toward Orion region. The colors correspond to the dust opacity derived from the Planck observations. The drapery pattern corresponds to the orientation of the magnetic field projected on the plane of the sky and integrated along the line of sight, as inferred from the Planck $353-\mathrm{GHz}$ observations. The yellow color between a declination of about $-5^{\circ}$ and $-10^{\circ}$ shows the Orion-A filamentary structure as illustrated in Fig. 2. The $\mathrm{B}_{\perp}$ lines on one side of this filament are mostly perpendicular to the filament (representing a small pitch angle for a helical morphology). While, on the other side, they are more parallel to the filament.

To find the $p$-values, we need the $\chi^{2}$ values and the degrees of freedom (DOF) of the model. For the helical model, we took one degree of freedom (i.e., $\left.B_{1} / B_{0}\right)$. For the first bow model, we took two degrees of freedom (i.e., $B_{0} / B_{1}$, and $\mathrm{R}^{\prime}$ ). For the second bow model, we took three degrees of freedom (i.e., $a_{\mathrm{R}}, b_{\mathrm{R}}$, and $\left.c_{\mathrm{R}}\right)$. Our best-fit bow model ( $\chi_{\text {tot }}^{2}=0.346$ with two DOFs) has a $p$-value of 0.84 . Our best helical model $\left(\chi_{\text {tot }}^{2}=0.310\right.$ with one DOF) has a $p$-value of 0.58 . Based on these $p$-values none of the models can be ruled out. It should be noted that the Reissl et al. (2018) bow model $\left(\chi_{\text {tot }}^{2}=0.872\right.$ with three DOF) has a $p$-value of 0.83 - similar to that of our bow model.

Therefore, it is likely that the bow morphology is more probable than both the helical or toroidal. Additionally, the results of the Monte Carlo analysis and our systematic bias investigation support our suggestion that the bow model is, overall, the most likely fit to the observed magnetic fields in the Orion-A filament.

Furthermore, as shown by the Planck results in Fig. 8, the $\mathrm{B}_{\perp}$ lines on one side of the Orion-A filament are mostly perpendicular to the filament (representing a small pitch angle for a helical morphology). While, on the other side, they are more parallel to the filament. Therefore, if we assume a helical magnetic field morphology for the Orion-A region, we would have to consider completely different pitch angles for the two sides of the filament, which is not sensible. Therefore, this simple visual analysis of the $\mathrm{B}_{\perp}$ orientation also seems to suggest that a bow morphology is a more natural fit to the data.

\subsection{Tilted models for Orion-A}

Finally, we briefly explored the effects of 3D geometry on our results. Recent observations by Großschedl et al. (2018) showed 
that the Orion-A filamentary structure is inclined along the LOS with respect to the observer. However, they showed that different parts of this region have different inclination angles. The part of the Orion-A region that contains Orion KL has a close-to-zero tilt. In particular, the region that contains L1641-N in Orion-A, which is very close to six of the seven data points taken in our analysis, has zero inclination in Fig. 3 of Großschedl et al. (2018). Due to this zero inclination at the region where our data points are accumulated, we suggest that we do not need to consider inclination of the Orion-A region along the line of sight for this study.

Consequently, the models of Eqs. (2), (4), and (5) represent a filament that is assumed to be lying in the plane of the sky without being tilted away from, or towards, the observer. With future data sets, where the data points are accumulated all along the Orion-A region, considering a more complex 3D geometry incorporating the study of Großschedl et al. (2018), is required. In that case, if one considers a tilted filament, the equations representing the models will not change, since the frame of reference is adjusted to the filament as illustrated in Fig. 3. However, when taking the average of each magnetic field component along the line of sight, one has to adjust $\mathrm{d} z$ in Eq. (7), to an extent that this $\mathrm{d} z$ is the line-of-sight path length from the observer's point of view and not in the filament's reference frame.

\section{Conclusions}

We present 3D magnetic field modeling around the Orion-A molecular cloud using existing dust polarization observations, which provide the plane-of-sky magnetic field, combined with the line-of-sight magnetic fields obtained by the method presented in Tahani et al. (2018). We first constructed models describing a toroidal, helical, and a bow-shape magnetic field morphology. While all three morphologies can explain the lineof-sight magnetic field reversal around Orion-A as observed by Tahani et al. (2018), we suggest that the bow morphology is the most natural magnetic field morphology for the Orion-A region.

This conclusion is based on the following arguments: (1) the bow model has the highest $p$-values; (2) our bow model seems to be the most consistent model with the observational data, and their uncertainty values in our Monte Carlo analysis approach, as well as with our investigation of systematic biases; (3) different $\mathrm{B}_{\perp}$ angles on the eastern side of the filament compared to the western side suggests that the data cannot be properly modeled by a single helix with the same pitch angle on both sides of the filament.

The analysis in this paper utilises the best rotation measure data currently available in the entire Orion-A region. As such, it is limited to the number of available RM sources in the Taylor et al. (2009) catalog, as well as the sensitivity of those observations. This analysis is also limited by the fact that the Planck maps provide only one value for $\mathrm{B}_{\perp}$ averaged across the entire region. Our results stress the need for more RM observations and higher-angular resolution dust polarization maps to further reconstruct the 3D magnetic field morphology.

Future RM catalogs from new generation surveys such as the Polarisation Sky Survey of the Universe's Magnetism (POSSUM) and the VLA sky survey (VLASS) should allow for the detection of a higher number of $B_{\|}$sources with better sensitivity. Thus, these new catalogs should provide bettersampled maps of the large-scale $B_{\|}$magnetic field with smaller errors. Combined with new and improved dust polarization maps (from instruments like APEX, ALMA, BLASTPOL2), future applications of the analyses presented in this work should allow us to map and model the 3D magnetic field geometry with better accuracy.

Acknowledgements. We thank the anonymous referee for his/her insightful comments that helped improve this paper. We used LATEX, Python and its associated libraries, Pycharm, and SAO Image DS9 for this work. We used Magnetar package $^{2}$ for line integral convolution (LIC) magnetic field visualization in Fig. 8. M.T. thanks Anahita Alavi for a discussion on statistical approaches. J.D.S acknowledges funding from the European Research Council under the Horizon 2020 Framework Program via the ERC Consolidator Grant CSF-648505. J.K. has received funding from the European Union's Horizon 2020 research and innovation program under grant agreement No 639459 (PROMISE). We acknowledge the Planck collaboration for the publicly-available data through the Planck Legacy Archive.

\section{References}

Arzoumanian, D., André, P., Didelon, P., et al. 2011, A\&A, 529, L6

Buckle, J. V., Davis, C. J., Francesco, J. D., et al. 2012, MNRAS, 422, 521

Chen, C.-Y., King, P. K., Li, Z.-Y., Fissel, L. M., \& Mazzei, R. R. 2019, MNRAS, 485, 3499

Contreras, Y., Rathborne, J., \& Garay, G. 2013, MNRAS, 433, 251

Crutcher, R. M. 1999, ApJ, 520, 706

Crutcher, R. M. 2012, ARA\&A, 50, 29

Crutcher, R. M., Wandelt, B., Heiles, C., Falgarone, E., \& Troland, T. H. 2010, ApJ, 725, 466

Fiege, J. D., \& Pudritz, R. E. 2000a, MNRAS, 311, 85

Fiege, J. D., \& Pudritz, R. E. 2000b, MNRAS, 311, 105

Gómez, G. C., Vázquez-Semadeni, E., \& Zamora-Avilés, M. 2018, MNRAS, 480, 2939

Goodman, A. A., Crutcher, R. M., Heiles, C., Myers, P. C., \& Troland, T. H. 1989, ApJ, 338, L61

Großschedl, J. E., Alves, J., Meingast, S., et al. 2018, A\&A, 619, A106

Hanawa, T., Nakamura, F., Matsumoto, T., et al. 1993, ApJ, 404, L83

Hartmann, L., Ballesteros-Paredes, J., \& Bergin, E. A. 2001, ApJ, 562, 852

Heiles, C. 1997, ApJS, 111, 245

Heiles, C., \& Robishaw, T. 2009, IAU Symp., 259, 579

Hennebelle, P., \& Falgarone, E. 2012, A\&ARv, 20, 55

Hennebelle, P., \& Inutsuka, S.-i. 2019, Front. Astron. Space Sci., 6, 5

Hildebrand, R. H., Kirby, L., Dotson, J. L., Houde, M., \& Vaillancourt, J. E. 2009, ApJ, 696, 567

Hoq, S., Clemens, D. P., Guzmán, A. E., \& Cashman, L. R. 2017, ApJ, 836, 199

Houde, M. 2011, ASP Conf. Ser, 449, 213

Houde, M., Bastien, P., Dotson, J. L., et al. 2002, ApJ, 569, 803

Inoue, T., Hennebelle, P., Fukui, Y., et al. 2018, PASJ, 70, S53

Johnstone, D., \& Bally, J. 1999, ApJ, 510, L49

Kainulainen, J., Beuther, H., Henning, T., \& Plume, R. 2009, A\&A, 508, L35

Li, H.-b., \& Houde, M. 2008, ApJ, 677, 1151

Li, H.-b., Fang, M., Henning, T., \& Kainulainen, J. 2013, MNRAS, 436, 3707

Liu, T., Li, P. S., Juvela, M., et al. 2018, ApJ, 859, 151

Matsumoto, T., Nakamura, F., \& Hanawa, T. 1994, PASJ, 46, 243

Matthews, B. C., Wilson, C. D., \& Fiege, J. D. 2001, ApJ, 562, 400

McKee, C. F., \& Ostriker, E. C. 2007, ARA\&A, 45, 565

Nakamura, F., Hanawa, T., \& Nakano, T. 1993, PASJ, 45, 551

Planck Collaboration XXXII. 2016, A\&A, 586, A135

Planck Collaboration XXXIII. 2016, A\&A, 586, A136

Planck Collaboration XXXV. 2016, A\&A, 586, A138

Reissl, S., Stutz, A. M., Brauer, R., et al. 2018, MNRAS, 481, 2507

Salji, C. J., Richer, J. S., Buckle, J. V., et al. 2015, MNRAS, 449, 1782

Schleicher, D. R. G., \& Stutz, A. M. 2018, MNRAS, 475, 121

Seifried, D., \& Walch, S. 2015, MNRAS, 452, 2410

Shibata, K., \& Matsumoto, R. 1991, Nature, 353, 633

Sivia, D., \& Skilling, J. 2006, Data Analysis A Bayesian Tutorial (Oxford: Oxford University Press)

Soler, J. D., Bracco, A., \& Pon, A. 2018, A\&A, 609, L3

Stutz, A. M., \& Gould, A. 2016, A\&A, 590, A2

Tahani, M., Plume, R., Brown, J. C., \& Kainulainen, J. 2018, A\&A, 614 A100

Taylor, A. R., Stil, J. M., \& Sunstrum, C. 2009, ApJ, 702, 1230

Tritsis, A., Panopoulou, G. V., Mouschovias, T. C., Tassis, K., \& Pavlidou, V. 2015, MNRAS, 451, 4384

Troland, T. H., \& Crutcher, R. M. 2008, ApJ, 680, 457

github.com/solerjuan/magnetar 